\title{
Cooling the vertical surface by conditionally single pulses
}

\author{
Pavel Karpov ${ }^{1, *}$, Alexander Nazarov ${ }^{1}$, Anatoly Serov ${ }^{1,2}$ and Victor Terekhov ${ }^{1,2}$ \\ ${ }^{1}$ Kutateladze Institute of Thermophysics SB RAS, Lavrentiev av. 1, Novosibirsk, 630090, Russia \\ ${ }^{2}$ Novosibirsk State Technical University, K. Marx av. 20, Novosibirsk, 630073, Russia
}

\begin{abstract}
You Sprays with periodic supply of the droplet phase have great opportunities to control the heat exchange processes. Varying pulse duration and frequency of their repetition, we can achieve the optimal conditions of evaporative cooling with minimization of the liquid flow rate. The paper presents experimental data on studying local heat transfer on a large subcooled surface, obtained on the original setup with multinozzle controlled system of impact irrigation by the gas-droplet flow. A contribution to intensification of the spray parameters (flow rate, pulse duration, repetition frequency) per a growth of integral heat transfer was studied. Data on instantaneous distribution of the heat flux value helped us to describe the processes occurring on the studied surface. These data could describe the regime of "island" film cooling.
\end{abstract}

\section{Introduction}

The current trend of developing miniature electronics dictates the demand for the new ways of removinghigh heat fluxes from small heat exchanging surfaces, and this can be ensured only by maintaining the admissible operating temperatures of equipment and instruments by the new active cooling systems.

Therefore, one of the most effective options for increasing heat transfer from selected power electronic components is controlled local cooling by the single pulses of a gasdroplet flow, working at film and evaporative cooling. The modern electronic components operate in temperature range of up to $100^{\circ} \mathrm{C}$, which defines the requirements for the working liquid flows (solid film, island coating, rivulets or drops).

It is known that when cooling the low-temperature surface, the temperature gradient at the liquid-solid surface interface plays a major role in heat transfer due to the Marangoni effect [1] because a significant contribution to heat transfer intensification is made by thermo-capillary convection due to evaporative cooling on a moving film - dry surface boundary.

According to the studies, development of island film cooling is observed on the surface, when tangential forces and local surface tension coefficient arise at insufficient wetting, resulting in shear film flows and dry spot formation, local film areas, rivulets and

${ }^{*}$ Corresponding author: Flags712008@yandex.ru 
droplets. The conditions of forming a thin evaporating film with microthickness along the contact line allow maintenanceof a high heat transfer rate.

\section{Experimental apparatus and instrumentation}

The experimental setup is a multifunctional device, consisting of: programmable multi-jet source of a pulsed gas-droplet flow (pulse injector), automatic calorimeter with removable heat exchanger, automated system of registering the gas-droplet flow parameters, including original capacitive sensors for detecting the thickness and velocity of waves on the liquid film, as well as gradient sensors of the local heat flux [2]. The applied integral method of heat transfer registration allowed us to record the dependence of heat transfer on the regime of gas-droplet flow formation, its intensity, characteristics of roughness the heat exchanging surface and other design features of the cooled body.

The controlled source of the gas-droplet jet is formed via the off-duty factor: droplet flow and continuous gas flow. The programmable multi-jet source of the pulsed gas-droplet flow is designed in the form of a two-chamber unit: for air and water. On the flat part of the source, there are 16 individually controllable liquid injectors in the form of $4 \times 4$ matrix with a spacing of $30 \mathrm{~mm}$. The liquid nozzle is a sprayer of four nozzles with $125-\mu \mathrm{m}$ diameter, switched-on by a solenoid valve. On the same surface, there are 25 gas nozzles with the outlet diameter of $0.35 \mathrm{~mm}$ around the liquid nozzle, which form the impinging multi-jet air flow. These investigation results relate to the regime, when the heat exchanger surface is normal to the gas-droplet flow and vertical relative to the horizon. The scheme of formation of the wall liquid and air flow is shown in Fig. 1.

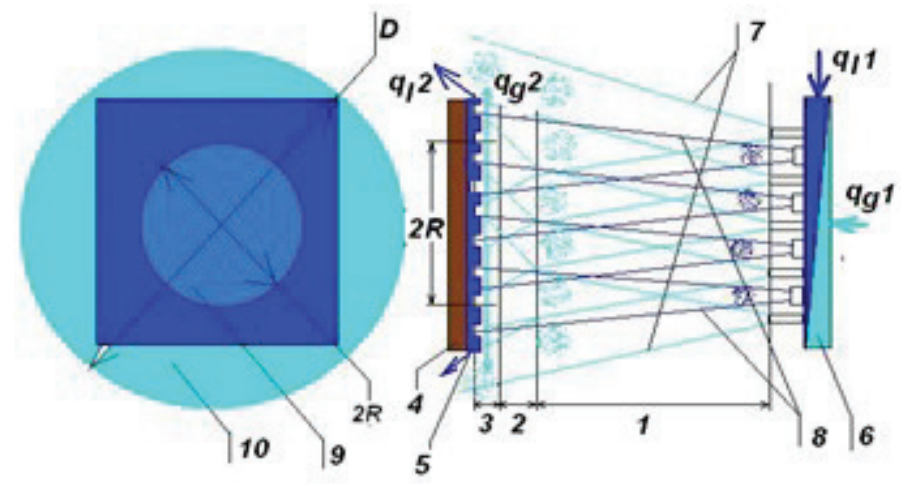

Fig. 1. Scheme of heat exchanger streamlining by the gas-droplet multijet flow.

$\mathrm{qg}^{1}$ - total mass flow rate of gas, $\mathrm{qg}^{2}$ - specific mass flow rate of gas on the heat exchanger surface,

$\mathrm{q}^{1}$ - total mass flow rate of liquid, $\mathrm{q1}^{2}$ - specific mass flow rate of liquid on the heat exchanger surface.

1-zone of submerged jets, 2- zone of gas jet turn, 3-zone of near-wall flows, 4-isothermal heat exchanger, 5 - near-wall liquid film, 6 - pulsed droplet and air injector, 7 - air jets, 8 - droplet jets.

Distilled water and water - ethanol mixture are used as the working liquids. To monitor the process of the near-wall film formation on the heat exchanger surface, we have determined the regime, when the impinging multi-jet gas-droplet flow forms a liquid film on the entire surface of heat exchanger. These regime allows observation of hydrodynamics and heat transfer in the central and peripheral areas of the near-wall flow of cooling liquid in the regime of conditionally single pulse, when the film with characteristic currents in specific zones of the surface is formed at the moment of irrigation by the droplet flow. The film evaporates partially or completely in-between the pulses. The above regime relates to cooling by conditionally single pulse. 


\section{Results and discussion}

This work is aimed at studying the processes of intensification of heat transfer from the heat-loaded surface at pulse irrigation by conditionally single multi-jet droplet flows. The short-term operation of all liquid nozzles with the ratio of period between pulses to duration of the droplet flow pulse higher than 20 is considered as a single pulse. The original programmable device forming the multi-jet pulse gas-droplet flows allows us to set the initial conditions of the cooling spray in a wide range.

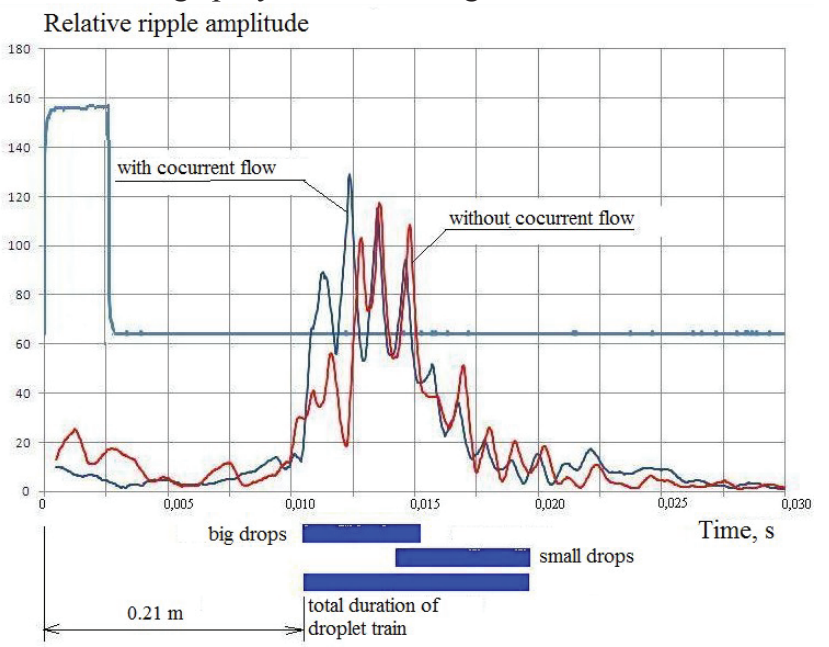

Fig. 2. Instantaneous value of droplet flow density near the heat exchanging surface.

Since formation of the multi-jet flame is studied insufficiently, at first, it was necessary to obtain data on distribution and behavior of the droplet train during target bombing. The effect of the co-current air flow on distribution of the droplet flow densityin the drift space and over the flow cross-section near the cooled surface was studied (Fig.2). The data showed that the droplet mixture flame in the co-current air flow has more uniform distribution of the droplet flow density over the cross-section and droplet grouping along the velocity vector, which result in more effective cooling ( 3 times). During the movement of a droplet train in the air flow, the large droplets group, forming a denser"head" of a pulse, which provides intensive "turbulization" of the formed film.

The next stage of research was data obtaining on the values of local and integral heat transfer coefficientsat irrigation by conditionally single pulses of the droplet-air mixture. Capabilities ofthe programmable multi-jet source allowed us to form a thin liquid film on the surface of the heat-loaded surface. The main condition was "conditional singlenessof a pulse". Fig. 3 shows the dependence of heat transfer obtained with the help of the local heat flux sensor. The peak corresponds to maximal heat transfer. When the bulk liquid is deposited on the surface, formed liquid film heating is observed (horizontal shelf), then there is uniform evaporation of the heated liquid film. The abrupt peak in the diagram corresponds byits time to the "end" of the droplet train. It is also possible to observe contribution of the secondary droplets to the process of heat entrainment from the surface. 


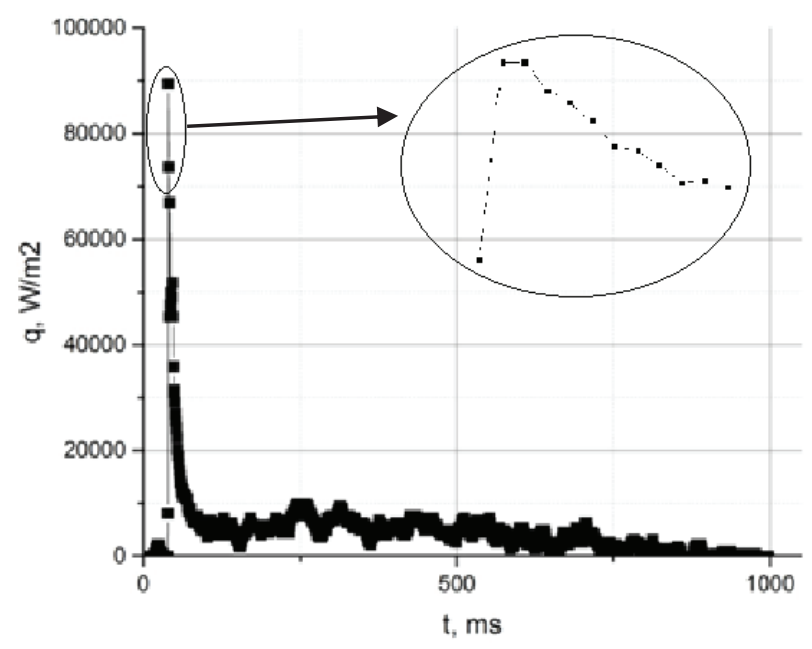

Fig. 3.Heatfluxdensityobtainedbythelocalheatfluxprobe

In all experiments, the pulse duration was constant and equal to $4 \mathrm{~ms}$. Repetition rate varied under the condition that the preceding pulse had no effect on the subsequent one (complete evaporation of all liquid film from the surface). The data on the values of heat transfer coefficients are shown in Table 1.

Table 1.Effectiveness of the spray

\begin{tabular}{|c|c|c|c|}
\hline $\mathbf{F , ~ H z}$ & $\mathbf{r , g} / \mathbf{( m 2 s )}$ & $\mathbf{h , W} / \mathbf{( m 2 K})$ & $\boldsymbol{\eta}$ \\
\hline 0.25 & 1.4 & 269.1 & 0.96 \\
\hline 1 & 3.6 & 446.8 & 0.87 \\
\hline 2 & 5.9 & 596.4 & 0.81 \\
\hline 4 & 10.7 & 795.5 & 0.75 \\
\hline 6 & 16.2 & 888.4 & 0.69 \\
\hline
\end{tabular}

The parameter, which characterizes the efficiency of the spray-system is its thermal efficiency; it equals the ratio of integral heat flux through the heat exchanger to the total value of system enthalpy [3]:

$$
\eta=\frac{q}{m \cdot\left[c_{\eta} \cdot\left(T_{w}-\tau_{s}\right)+r\right]}
$$

where - is het flux density $\left(\mathrm{W} / \mathrm{m}^{2}\right), \mathrm{m}$ - is specific mass flow rate $\left(\mathrm{kg} / \mathrm{m}^{2} \mathrm{~s}\right), \mathrm{C}_{\mathrm{p}}$ - is specific heat capacity $(\mathrm{J} / \mathrm{kgK}), \mathrm{w}, \mathrm{s}-$ are temperatures of the heat-exchanging surface and liquid $(\mathrm{K}), \mathrm{r}-$ is latent heat of vaporization of liquid phase $(\mathrm{J} / \mathrm{kg})$. DataintheTableshowthat at frequency $\mathrm{F}=0.25 \mathrm{~Hz}$, the most effective heat removal is observed. 


\section{Conclusions}

The investigations have shown that it is possible to construct the compact efficient cooling systems for electronic devices, which ensure effective cooling at low coolant flow rate via the regime, controlled by coordinate and time, combining film and evaporative cooling.

The work was financially supported by RFBR (project 16-38-00853).

The work was financially supported by the grant of Russian President NSh-8780.2016.8

\section{References}

1. E. Gatapova, O. Kabov, I. Marchuk, Technical Physics Letters, 30, 418 - 421, (2004)

2. A. Nazarov, A.Serov, M. Bodrov, Technical Physics, 80,724-727, (2010)

3. C. Pedersen, Int. J. Heat Mass Transfer,13, 369-381, (1970) 